\title{
Dynamics of primordial binary stars in multiple-population globular clusters
}

\author{
Jongsuk Hong ${ }^{1}$, Enrico Vesperini ${ }^{1}$, Antonio Sollima ${ }^{2}$, Steve \\ McMillan ${ }^{3}$, Franca D'Antona ${ }^{4}$ and Annibale D'Ercole ${ }^{2}$ \\ ${ }^{1}$ Department of Astronomy, Indiana University, Bloomington, IN, 47401, USA \\ ${ }^{2}$ INAF - Osservatorio Astronomico di Bologna, via Ranzani 1, I-40127 Bologna, Italy \\ ${ }^{3}$ Department of Physics, Drexel University, Philadelphia, PA 19104, USA \\ ${ }^{4}$ INAF - Osservatorio Astronomico di Roma, via di Frascati 33, I-00040 Monteporzio, Italy
}

\begin{abstract}
We have performed a survey of $N$-body simulations to explore the dynamics of primordial binaries in multiple-population globular clusters. We show that, as a consequence of the initial differences between the spatial distribution of first-generation (FG) and secondgeneration (SG) stars, SG binaries are disrupted more efficiently than FG binaries. The effects of dynamical evolution on the surviving binaries produces a difference between the SG and the FG binary binding energy distribution with the SG population characterized by a larger fraction of high binding energy (more bound) binaries. We also explore the evolution of the radial variation of the SG-to-FG binary number ratio and find that although the global binary fraction decreases more rapidly for the SG population, the local binary fraction measured in the cluster inner regions may still be dominated by SG binaries.
\end{abstract}

Keywords. globular clusters : general - stars : chemically peculiar

\section{Introduction}

Theoretical models for the formation of multiple-population globular clusters predict that second-generation (SG) stars form in a compact subsystem in the cluster central regions (see e.g. D'Ercole et al. 2008; Bekki 2011) and several observational studies have indeed found clusters in which SG stars are more centrally concentrated than the firstgeneration (FG) population (see e.g. Sollima et al. 2007; Bellini et al. 2009; Lardo et al. 2011). In a previous study (Vesperini et al. 2011), we have shown that the initial differences in the structural properties of FG and SG stars can have significant implications for the evolution of the binary star population. We present here a brief summary of the results of an extended survey of $N$-body simulations aimed at exploring the evolution of binary stars in multiple-population globular clusters (see Hong et al. 2015 for a complete presentation of our results).

\section{Results}

We have followed the evolution of systems with different structural parameters, binary fraction, and binary binding energy. In Fig.1 we show the results of two of the simulations of our survey. For the results shown in Fig. 1, the initial multiple-population system is characterized by a ratio of the FG to the SG half-mass radius equal to 5 and an initial binary fraction equal to 10 per cent. We compare the results of our simulation of the multiple-population cluster with those of simulations of a single-population cluster with the same total mass and radius. We present here the results for two values of the binary binding energy corresponding to values of the hardness parameter (as measured in the reference single-population cluster) equal to $x_{\mathrm{g}, 0}=5$ and $x_{\mathrm{g}, 0}=20$. 

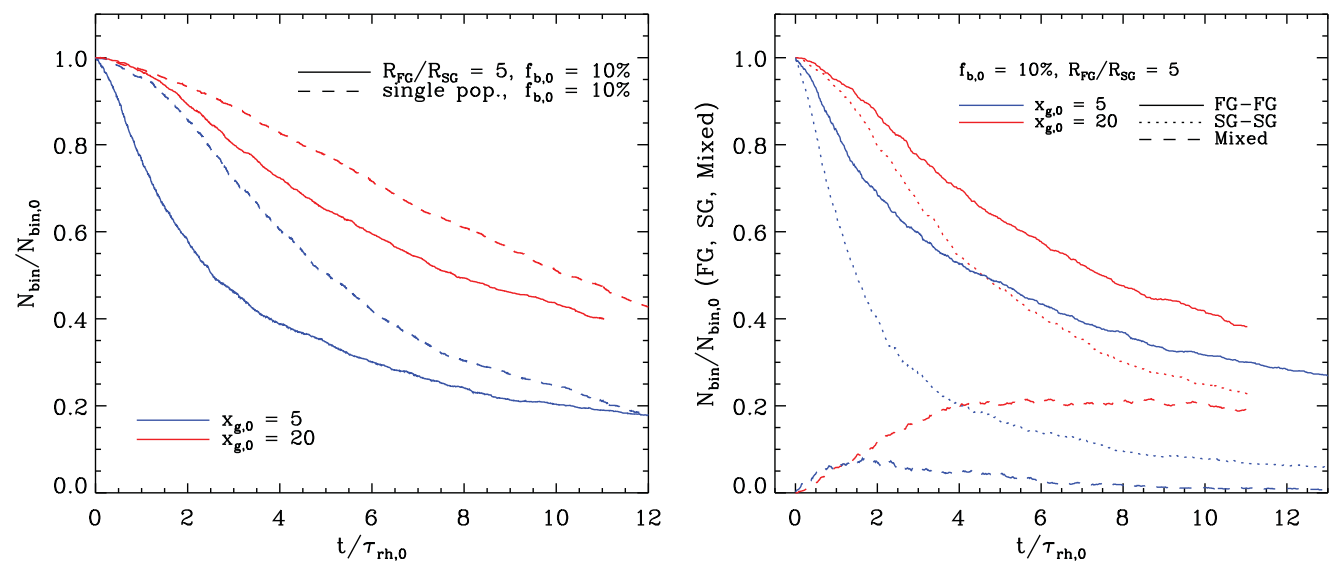

Figure 1. Left Time evolution (time is normalized to the initial half-mass relaxation time) of the total number of binaries (normalized to the initial total number of binaries) for multiple-population and single-population systems for binaries with different initial hardness. Right Time evolution of the number of FG, SG and mixed binaries (binaries with one FG and one SG component) for a multiple-population cluster and binaries with different initial hardness parameter.

The two panels of Fig.1 illustrate some of the general conclusions reached in our investigation. In particular the left panel shows that binary disruption is in general enhanced in multiple-population clusters compared to single-population systems. The right panel shows the preferential disruption of SG binaries. This is a consequence of the fact that the SG population is more spatially concentrated than the FG population.

Some binaries are not disrupted and can undergo an exchange event during which mixed binaries composed of one FG and one SG component are produced.

Although SG binaries are disrupted at a larger rate than FG binaries and the global number of FG binaries is always larger than that of SG binaries, the local binary fraction in the cluster inner regions may be larger for SG binaries. We have shown that the SG-to-FG binary number ratio measured at a distance from the cluster center equal to about $1-2.5$ times $r_{\mathrm{h}}$ (where $r_{\mathrm{h}}$ is the cluster half-mass radius) is approximately equal to the global value of this ratio. Our simulations also show that the effects of dynamical evolution produce a difference between the SG and the FG binary binding energy distribution of the surviving binaries with the SG population characterized by a larger fraction of high binding energy (more bound) binaries.

\section{Acknowledgements}

JH acknowledges support from AAS International Travel Grant.

\section{References}

Bekki, K., 2011, MNRAS, 412, 2241

Bellini, A., Piotto, G., Bedin, L. R., et al. 2009, A\&A, 507, 1393

D'Ercole, A., Vesperini, E., D'Antona, F., McMillan, S. L. W., \& Recchi, S., 2008, MNRAS, 391,825

Hong, J. Vesperini, E., Sollima, A., McMillan, S. L. W., D’Antona, F., \& D’Ercole, A., 2015, submitted to MNRAS

Lardo, C., Bellazzini, M., Pancino, E., et al. 2011, A\&SA, 525, AA114

Sollima, A., Ferraro, F. R., Bellazzini, M., et al. 2007, ApJ, 654, 915

Vesperini, E., McMillan, S. L. W., D'Antona, F., \& D'Ercole, A., 2011, MNRAS, 416, 355 